\title{
A Survey on Social Circle Influenced Personalized Recommendation System
}

\author{
Vishal J. Kadam ${ }^{1}$, Vilas S. Gaikwad ${ }^{2}$ \\ 1,2 Department of Computer Engineering, JSPM Narhe Technical Campus, Savitribai Phule Pune University, Pune, Maharashtra, India
}

\begin{abstract}
In contemporary world, social networking sites are becoming an inseparable part of everyone's life. With the advent and affection of online social networks, the social network based approach to recommendation has emerged. Recommender systems are the tools of choice to choose online information relevant to a given user. Users are interested to share their experiences through ratings, reviews, polls, blogs etc. which assists to recommend the items of user interest. Rapid growth of information generated by online social networks leads to increase in demand of efficient and effective recommender systems to give accurate results. Traditional recommendation techniques are limited because they do not consider factors of social relation in the social network for giving recommendation. The new intrinsic parameters of social network like personal interest, interpersonal interest similarities and interpersonal influence bring opportunities and challenges for recommender system to solve the cold start and sparsity problem of datasets more efficiently. This survey paper is to study various traditional recommendation techniques and main three social aspects, and how these factors are to be fused into a personalized recommendation model to give efficient recommendations to the user.
\end{abstract}

Keywords: Social Circle, Personalized Recommendation System, Interpersonal Influence, Matrix Factorization, Association Rule

\section{Introduction}

Recommendation system (RS) has been successfully used to solve problem overwhelming. Social networks such as twitter, facebook etc. are handling large scale of information by recommending user interested items and products. RS has wide range of applications such as new social tags, movies, research articles, music etc. According to the user input and different attribute items can be recommended, which is closely related to user interest.

Survey shows that more than 25 percent of sales generated through recommendation. Over $90 \%$ peoples believe that products recommended by friend are useful [1] and 50\% people buy the recommended products or items of their interest. Google+ introduced "Friends Circle" to filter the contacts according to different activities and strategies [2], which helps users to be closer to their friends. In a large web space, recommendation helps to find items of user interest [7]. Collaborative filtering and content based filtering are widely used methodologies for recommendation [3]. For Data Mining works cold start has been a serious problem. Even though we have many algorithms to work on Data Mining, cold start has made people to step back in analyzing the functionality of those algorithms lead to little decrease in creativity and optimizations in data mining algorithms[4][5]. Cold start can be described as unavailability of data for modelling algorithms [7]. Web is always dynamic, so it very difficult to predict the user interested items [14] in time.

Personalized RS constitutes factors such as interpersonal interest, person's interest and interpersonal influence [1]. Personalized RS is helpful to recommend the items on social networks with the aim that recommended items should base on their historical behavior and interpersonal relationship of social networks. The increasingly popular online social networks provide additional information to enhance pure rating-based RS [2]. Recommendation in traditional system focuses on pair of (buyer, item) whereas social recommendation focuses on triplet (seller, buyer, item) which enhances the more appropriate items of user interest [10]. The quality of the recommendation can be achieved with the help of user interpersonal interest in social network [16]. Several social-trust based RS have recently been proposed to improve recommendation accuracy. The interpersonal relationship in the friend's circle of social networks and social contexts [20] helps to solve cold start and sparsity problem.

\section{Literature Survey}

Phelan et al. [14] proposed a news recommendation technique utilizing real-time Twitter data as the basis for ranking and recommending articles from a collection of really simple syndication feeds. And one of the conclusions is that users with more friends tend to benefit more.

Yuan et al. have explored a kind of social relation, the membership, and its combined effect with friendship [24]. The two types of heterogeneous social relations are fused into the Collaborative Filtering based recommender via a factorization process. And the distinguished effectiveness of social relationships in the sparse data condition was demonstrated.

Java et al. [25] had analyzed a large social network in a new form of social media known as micro-blogging. It has a high degree correlation and reciprocity, indicating close mutual acquaintances among users. They had identified different types of user intentions and studied the community structures. Categorizing friends into groups (e.g. family, co-workers) would greatly benefit the adoption of micro-blogging platforms to analyze user intentions. That is to say user intentions or interests can be reflected by those of its friends.

Yang et al. [2] propose to use the concept of ,inferred trust circle $^{\text {ee }}$ based on the domain-obvious circles of friends on social networks to recommend user favorite items. Their 


\section{International Journal of Science and Research (IJSR) \\ ISSN (Online): 2319-7064}

Index Copernicus Value (2013): 6.14 | Impact Factor (2014): 5.611

approach not only refines the interpersonal trust in the complex networks, but also reduces the load of big data.

Meanwhile, besides the interpersonal influence, Jiang et al. [3] demonstrate that individual preference is also a significant factor in social network. Just like the idea of interpersonal influence, due to the preference similarity, user latent features should be similar to his/her friends" ${ }^{\text {ee }}$ based on the probabilistic matrix factorization model. However, do all users actually need the relationship on the social networks to recommend items? Does the relationship submerge user's personality, especially for the experienced users? It is still a great challenge to embody user's personality in RS, and it is still an open issue that how to make the social factors be effectively integrated in recommendation model to improve the accuracy of RS.

\section{Research Challenges}

\subsection{Cold Start Problem}

Cold start problem refers to the situation when a new user or items newly added to the system [7]. With rapid increasing of registered users various products, the problem of cold start occur [8]. Three kinds of cold start problems are: new user problem, new item problem and new system problem [8]. In such cases, it is very difficult to provide recommendation, as in case of new user, there is very less information about user that is available. For a new item, no ratings are usually available and thus collaborative filtering cannot make useful recommendations in case of new item as well as new user. For the new system, it is difficult to find the pattern [6] as there is very less information about user and newly added product or items. Most important factor in social network is individual preferences and interpersonal relations such as "friend circle" [1] helps to solve the cold start problem.

\subsection{Sparsity Problem}

Sparsity problem is one of the major problems encountered by recommender system is data sparsity has great influence on the quality [21] of recommendation. The main reason behind data sparsity is that most users do not rate most of the items and the available ratings are usually sparse. In collaborative filtering [22] technique it is important that the more users are required to be rated the item. Though high rating [6] given by few users leads to problem of sparsity. To prevail over the sparsity problem, one can use user profile information [6] while calculating user similarity item with others. Similarity in users can be identified with the aid of age, area code, gender, demographic segment etc. Sparsity problem also resolved by associative retrieval framework and related spreading activation algorithms [10]. Sparse rating matrix can used to resolve the sparsity problem. Item based mining and associative retrieval technique [5] also used to overcome the problem of sparsity.

\subsection{Overspecialization}

It prevents user from discovering new items and other available options [6]. However, diversity of recommendations is a desirable feature [7] [8] [22] of all recommendation system. After solving the problem using genetic algorithms, user will be provided with a set of different and a wide range [6] of alternatives Overspecialization occurs if system recommends only items which having high rating against the user profile. For example if a person has no experience with chinese foods will never receive recommendation [22] though the Chinese restaurant are available in the city. Similarity fusion and probabilistic fusion [12] are used to solve the problem of overspecialization. Out-of-Box method [18] can be used to resolve the overspecialization problem. Similarity measures, sampling and dimension reduction in collaborative filtering [15] helps to overcome the overspecialization problem.

\subsection{Scalability}

Scalability is the property of system indicates its talent to handle emergent amount of information [6] in a stylish manner. With vast growth in information over internet, it is obvious that the recommender systems are having an explosion of data [10] and thus it is a big dispute to handle with continuously growing demand. Some of the recommender system algorithms deal with the computations, which increase with growing number of users and items. Method projected for treatment scalability problems are based on approximation mechanism [22].

Even if they advance routine, most of the time they result in correctness reduction. Scalability problem can be resolved by pre-processing, clustering and user based collaborative filtering [5] [6] [16]. In pre-processing noisy data is cleaned and transformed [12] to the recommendation system. Clustering helps to group the similar items [13] [5] with intra cluster distance as small as possible and inter cluster distance as large as possible. Used based collaborative filtering uses the person's profile. Personalized recommendation algorithm [1] [3] also used to overcome the problem of scalability. It is also overcome by using association retrieval [23].

\section{Social Circle Influenced Personalized Recommendation System Approaches In Social Network}

For General Recommendation most popular methods are content based filtering and item based filtering. Both of these systems are vulnerable to cold start and sparsity problem. To overcome these problems personalized recommendation system uses the interpersonal interest, social profile etc. to recommend user interested items.

Peoples are more likely to buy products recommended by their friends, so personalized recommendation systems helps to make decision. Some of the personalized recommendation system approaches are discussed as below;

\subsection{Basic Matrix Factorization}

The task of RS is to decrease the error of predicted value to the real rating value. Thus, the BaseMF model is trained [1] [5] on the observed rating data by minimizing the objective 


\section{International Journal of Science and Research (IJSR) \\ ISSN (Online): 2319-7064}

Index Copernicus Value (2013): 6.14 | Impact Factor (2014): 5.611

function. This is based on the probabilistic matrix factorization [4], which uses the low rank matrix. The idea of matrix factorization is to decompose a matrix $\mathrm{M}$ into the product of several factor matrices, i.e. $M=F 1 F 2 \ldots F n$ [5] where $\mathrm{n}$ can be any number, but it is usually 2 or 3 . Base matrix factorization has gained popularity over several years due to its superior performance in scalability [8] [10].

Whenever connection between variable and observed variable is estimated during the training recommendation can made by computing possible interaction [18] with each product in separate matrix, called base matrices. Basic matrix factorization is combined with the social network data [2] in recommendation system.

\subsection{ContextMF Model}

Jiang et al. [3] demonstrate the significance of social contextual factors (including interpersonal influence and individual preference) for item adopting on real Facebook and Twitter style datasets. The task of ContextMF model in [3] is to recommend acceptable items from sender to receiver. Here, the factor of interpersonal influence is similar to the trust values in CircleCon model [2].

Moreover, individual preference is mined from receiver's historical adopted items. User rated items has more influence than individual preference in ContextMF model, because it easier for the recommended items of our model to be transformed into purchase rate than the adopted items [11] in Facebook style social networks. It is observed that neighbors in the social network have similar interest, Context matrix factorization helps to identify similar interest by training objective function [2]. Direct neighbors can be identified by Bayesian inference [4] which helps to identify user personal interest directly related [1] to rated items. Individual interest and inter personal interest are considered in context matrix factorization because it is easy to recommend the user interested items in real time. The performance of the cold start is improved [4] by 50\% using ContextMF.

\subsection{CircleCon Model}

The CircleCon model [2] has been found to outperform BaseMF and SocialMF [4] with respect to accuracy of the RS. The approach focuses on the factor of interpersonal trust in social network and infers the trust circle. The trust value of user-user is represented by the matrix.

Furthermore, the whole trust relationship in social network is divided into several sub-networks, called inferred circle and each circle is related to a single category of items. CircleCon model can be processed in the following steps;

First, trust circle inference, this can be identified with the aid of different genre (categories) with certain threshold value. Second, trust value assignment, this can be done with equal trust, enterprise based trust and trust splitting. Social contexts integrated [3] to give more accurate results. In a large scale social network rating and review is important, flixster [4] uses this model to recommend the movies to other users based on the user profile. They allow the users to rate and review the movies based on their interest, and collects the information. CircleCon model gives the high quality [11] of recommendation in social network over large data sets.

\section{Conclusion}

This paper surveys the different methodologies used to build recommendation system in social network with user interest. Here introduced the basic of the recommendation system approaches and research challenges. Described different methodologies used to build efficient recommendation system along with the user's personal interest and interpersonal influence on social network by base matrix and social matrix factorization.

\section{References}

[1] X Qian, H Feng, $\mathrm{G}$ Zhao, $\mathrm{T}$ Mei "Personalized Recommendation Combining User Interest and Social Circle", Knowledge and Data Engineering, IEEE Transactions, 2014.

[2] X. -W. Yang, H. Steck, and Y. Liu. "Circle-based recommendation in online social networks". KDD"12, pp. 1267-1275, Aug.2012.

[3] M. Jiang, P. Cui, R. Liu, Q. Yang, F. Wang, W. -W. Zhu and S. -Q. Yang. "Social contextual recommendation". CIKM"e12, pp. 45-54, 2012.

[4] M. Jamali and M. Ester. "A matrix factorization technique with trust propagation for recommendation in social networks". In Proc. ACM conference on Recommender systems (RecSys), 2010.

[5] R. Salakhutdinov and A. Mnih. "Probabilistic matrix factorization". In NIPS 2008, 2008.

[6] G. Adomavicius, and A. Tuzhilin. "Toward the next generation of recommender systems: a survey of the state-of-the-art and possible extensions". Knowledge and Data Engineering, IEEE Transactions on, pp. 734-749, Jun. 2005.

[7] M. Jahrer, A. Toscher, and R. Legenstein. "Combining predictions for accurate recommender systems". KDD'10, pp. 693-702, 2010.

[8] H. Ma, I. King, and M. R. Lyu. "Learning to recommend with social trust ensemble". SIGIR'09, 2009.

[9] X. Yang, Y. Guo and Y. Liu. "Bayesian-inference based recommendation in online social networks". The 30th Annual IEEE International Conference on Computer Communications (INFOCOM), 2011.

[10] J. Huang, X. Cheng, J. Guo, H. Shen, and K. Yang. "Social recommendation with interpersonal influence". In Proceedings of the 19th European Conference on Artificial Intelligence (ECAI), pp.601-606, 2010.

[11]H. Ma, H. Yang, M.R. Lyu, and I. King. "Sorec: Social recommendation using probabilistic matrix factorization". CIKM $08,2008$.

[12] J. Wang, A.P. de Vries, and M.J.T. Reinders. "Unifying user-based and item-based collaborative filtering approaches by similarity fusion". SIGIR'06, 2006.

[13]P. Cui, F. Wang, S. Liu, M. Ou, S. Yang, and L. Sun. "Who should share what? item-level social influence prediction for users and posts ranking". SIGIR, pp. 185194, 2011

\section{Volume 4 Issue 11, November 2015}


[14]H.R. Kim and P.K. Chan. "Learning Implicit User Interest Hierarchy for Context in Personalization". In Proc. of International Conference on Intelligent User Interface, 2003.

[15] O.Phelan, K. McCarthy, and B. Smyth. "Using twitter to recommend realtime topical news." In Proceedings of the third ACM conference on Recommender systems, pp. 385-388. ACM, 2009.

[16]R. Sinha, and K. Swearingen, "Comparing recommendations made by online systems and friends". Dublin, Ireland, 2001.

[17] A. Rahman, and S. Hailes, "Supporting trust in virtual communities". In Proceedings of the 33rd Hawaii International Conference on System Sciences. Maui, HW, USA (2000).

[18]H Ma, D Zhou, C Liu, MR Lyu, I King. "Recommender Systems with Social Regularization". Fourth ACM international conference on Web search and data mining, 2011.

[19]A Bellogín, P Castells, I Cantador. "Self-adjusting Hybrid Recommenders Based on Social Network Analysis". $34^{\text {th }}$ international ACM SIGIR conference on Research and development in Information Retrieval, 2011.

[20]Xavier Amatriain "Mining Large Streams of User Data for Personalized Recommendations" ACM SIGKDD Explorations, 2012.

[21]L Sharma, A Gera "A Survey of Recommendation System: Research Challenges" International Journal of Engineering Trends and Technology, 2013

[22]GS Sadasivam "A Survey on Personalized Recommendation Techniques"

[23] Y Chen, C Wu, M Xie, X Guo "Solving the Sparsity Problem in Recommender Systems Using Association Retrieval", Journal of computers, 2011

[24]Q. Yuan, L. Chen, and S. Zhao, "Factorization vs. regularization: Fusing heterogeneous social relationships in top-N recommendation," in Proc. 5th ACM Conf. Recommender Systems, Chicago, IL, USA, 2011.

[25] A. Java, X. Song, T. Finin, and B. Tseng, "Why we twitter: Understanding microblogging usage and communities," in Proc. 9th WebKDD, 1st SNA-KDD, San Jose, CA, USA, 2007, pp. 56-65. 